\title{
CONSIDERACIONES SOBRE LA ESTRATIFICACIÓN SOCIAL A NIVEL HOSPITALARIO (EL. MÉDICO Y LA ENFERMERA)
}

\author{
Alicia E. Kaufmann \\ (Universidad de Barcelona)
Elvira Guilera
(Coordinadora del Programa de Gestion
Hospitalaria en el ESADE)

El presente trabajo constituye un intento de analizar la estratificación social que se produce a nivel hospitalatio y que condiciona su gestión. Para ello, definimos en términos generales los rasgos principales de la medicina bajo el capitalismo, el lugar que en él ocupa el sector sanitario, las características de los hospitales y las profesiones consideradas, es decir, el médico y la enfermera, así como también hechos pasados y presentes que configuran la imagen de las mismas. Concedemos particular importancia al elemento técnico que, al ser muy diferenciado, refuerza la desigual distribución de autoridad entre ambos, y que amén de otras variables, sienta las bases del tipo de estratificación existente. 
La elección de este tema no es casual sino que obedece a una serie de factores que confluyen transformando en costosa y conflictiva la gestión sanitaria.

Estos factores detivan fundamentalmente del auge creciente de la población activa empleada en el sector sanitario, que en España, al igual que en Estados Unidos, constituye el segundo sector que más capital inumano ocupa después de educación, del peso económico de este factor y de las características socio-profesionales de quienes componen este área.

Evidentemente, no se trata de un fenómeno privativo de nuestro país, sino que es la tendencia que siguen, tanto en los países centrales como los que de ellos dependen, habida cuenta de las diferencias entre cada uno de ellos considerando el grado de desarrollo alcanzado, y la posición desventa. josa que ocupa el sector sanitario en las economías del sistema capitalista, particularmente si se trata de países independientes.

Las cifras que se destinan a la financiación global del sector oscilan entre el 5 y el $8-10 \%$ del PNB. Recientemente, en la reunión de ministros de Sanidad en los países del MCE, se acordó no sobrepasar la cifra del $10 \%$ como porción máxima del PNB a destinar a Sartidad, y de esta importante cifra, entre un 6 y un $8 \%$ se dirige a la financiación de los hospitales, que se transforman así en los más importantes consumidores del presupuesto sanitario. De los costes de los hospitales, entre el 50 y el $80 \%$ corresponde a salatios y conceptos de coste de personal.

El ámbito de estudio será entonces el moderno hospital general, que en las sociedades avanzadas se erige en la base de la estructura sanitaria y que constituye el contexto en donde ambas profesiones entran en interacción. Partimos del supuesto que la gestión de los hospitales está muy marcada por el papel y la posición que dentro de ellos asume el estamento médico, no sin antes considerar la distribución de las grandes profesiones y la consiguiente autoridad en el seno del hospital de los grupos implicados en el proceso. 
En cuanto a la estratificación profesional en los hospitales españoles, los grandes grupos profesionales se distribuyen como sigue:

- Médicos y personal titulado con grado supetior - $25 \%$

- Enfermeras y personal titulado con grado medio. $30 \%$

_ Personal auxiliar .................................... $15 \%$

- Administrativos .................................... $9 \%$

- Personal de oficios ............................... $9 \%$

- No cualificado .................................... $12 \%$

Los datos" registran una preponderancia marcada de dos profesiones - los médicos y las enfermeras -que totalizan el $55 \%$ del total del personal. Este último extremo, entre otras significaciones, daría una de las más características, que es la elevada cualificación del personal en los hospitales, factor que condiciona una determinada problemática de gestión.

Por lo tanto, el estudio del tema obliga al planteamiento de dos opciones metodológicas. El hacerlo con toda su amplitud (tarea ingente y que desbordaría la intencionalidad de este trabajo) o el acotarlo lo más posible a los efectos de realizar un trabajo concreto en profundidad.

Adoptada esta segunda opción, aludimos en este trabajo al sistema de estratificación que se da en el sector sanitario analizando en el mismo tan sólo las profesiones que son especificas del sector y que condicionan la gestión de los hospitales con mayor peso; es decir, los médicos y las enfermeras, no sin antes aludir brevemente a las características del modo de producción capitalista y su relación e influencias en el sector sanitatio, por lo cual primero definiremos el todo para luego entender cada una de sus partes entrelazadas, es decir, que la medicina obedece a las mismas fuerzas que determinan a la sociedad en su conjunto. ${ }^{2}$

En el modo de producción capitalista la medicina se articula con el proceso de producción económica, el trabajador pierde el control y la propiedad de los medios de trabajo y tiene en consecuencia que vender su fuerza de trabajo para sobrevivir. La medicina se define, en este modo de producción, como la actividad cuyo objeto primordial es la conservación y adaptación de la fuerza de trabajo. Jean Claude Polack ${ }^{3}$ expresa esta idea de la siguiente forma:

1. Asociación Española de Administración de Hospitales, «Encuesta realizada en 30 centros hospitalarios de más de 450 camas», documento de đivuligación interna.

2. Vicente Navarto, La medicina bajo el capitalismo (Barcelona: Crítica, 1978).

3. Jean Claude Polack, La medicine du capital (París: Maspéro, 1972). 
«La atención (médica) no es un producto, un objeto inseparable de su agente, una mercancía susceptible de circular como auténtico valor de cambio. Este producto no desaparece al ser consumido. Cuando se dirige a la fuerza de trabajo el acto terapéutico eleva el nivel de esa fuerza de trabajo o contribuye a su mantenimiento en el cuadro de la reproducción. Al contribuir a elevar el nivel de la productividad, la atención es indirectamente productiva. [...] Esta nosología ambigua no permite confundir la salud con el sector terciario, o con un proceso de comercialización, de publicidad o de servicio. La medicina no tiene especialmente el dominio de los cuadros administrativos de la economía, sino a la definición permanente de un nivel de productividad. Las fuerzas productivas son su objeto elegido. Es relativamente direc$\mathrm{ta}$, inmediata por la misma influencia de la actividad de salud sobre la marcha de la economía. El sistema de atención tiene bajo su control la parte humana de la producción, el trabajo.»

Al respecto otro autor, F. Steudier, señala:

«La evolución del sistema de salud parece estar regida por las exigencias propias de la estructura económica y por la relación de fuerzas que se establece entre los distintos grupos sociales (partidos políticos, sindicatos, distintos grupos de presión) y el Estado para obtener mejoras en materia de salud.» ${ }^{4}$

Es así como la medicina, en el modo de producción capitalista, no sólo se presenta como reguladora de la productividad de la fuerza de trabajo, sino que también juega un papel importante en la disminución de las tensiones, producto de la desigualdad social. Se transforma el lenguaje médico y surge una nueva idea, la del precio de la vida y el costo que entraña su protección.

Es a nivel hospitalatio donde se visualiza con mayor claridad este nuevo papel asignado a la medicina, como así también el tipo de telaciones que se establecen entre los grupos profesionales. En la actualidad el moderno hospital constituye una organización compleja con una jerarquía de status, roles, derechos y obligaciones. Existe un doble sistema de autoridad dado por una autoridad administrativa y una autoridad médica, que a menudo entran en conflicto por su competencia por el poder. La división del trabajo es elevada y es una consecuencia directa del nivel tecnológico,

4. François Steudler, Sociologie medicale (París: Armand Colin, 1972). 
que hace que el funcionamiento del hospital sea análogo al de una gran empresa, en donde cada miembro debería desarrollar una función específica. A nivel del tratamiento del individuo, esto se traduce en un tratamiento fragmentario, con un grado de despersonalización creciente, en donde el paciente pasa a ser un número, un objeto con una serie de síntomas orgánicos. Esta creciente especialización no sólo produce problemas de comunicación entre el personal y los pacientes, sino entre los miembros del personal.

Así, en el hospital, la acción fundamental, la de la devolución de la salud, gira en torno a dos profesiones básicas: el médico y la enfermera.

La moderna concepción de las formas de llevar a cabo el proceso asistencial nos fija unos roles dentro de este proceso para cada uno de estos profesionales. El médico es quien pone uen marcha» el hospital. Pero este papel debe tener un inmediato apoyo en el que lleva a cabo la enfermería.

Uno con el otro se complementan y, aunque es cierto que el paciente sin la actuación del médico no podría avanzar en su proceso, también lo es que eI médico sin la enfermería no podría concluir el proceso.

Si bien para el funcionamiento del hospital ambos roles son funda mentales, existe una desproporción de autoridad, al punto de poder argüir que la relación entre ambos es más bien de dominación/subordinación que de colaboración. Explicamos este hecho a través de una serie de factores, siendo nuestra hipótesis inicial que esta situación surge como emergente de una distribución diferencial del conocimiento, diferencias de sexo y de clase social. El grado de influencia de cada uno de estos factores también será tema de análisis.

La segunda hipótesis a confirmar alude al hecho de que la estructuración del conocimiento de estas profesiones es productora y refuerza las desigualdades sociales en el campo de la sanidad y por ende reproduce la estructura de clases existente.

Algunas de estas diferencias ya vienen dadas históricamente en función del grupo profesional al que cada uno de ellos pertenece. En cuanto al médico, y siguiendo las ideas de Steudler,, podemos decir que el médico pertenece a un grupo profesional específico, su conducta está regida por una setie de normas particalares y la educación que recibe le confiere una cierta imagen de la sociedad y de los enfermos. En las sociedades occidentales los médicos tienen un elevado status, debido a que su imagen está asociada con conocimientos, poder y dedicación. El sitio elevado que ocupa en la escala de estratificación no sólo proviene de sus ingresos, sino del poder que le confere su posición. Participa en situaciones de crisis

\section{Ibid.}


y su poder es ambivalente en función del poder que ejerce sobre la vida y la muerte. Así, los médicos aparecen en posición de superioridad, no sólo frente a los pacientes, sino también al personal subordinado.

Esta superioridad está otorgada por una autoridad carismática por el predominio de sus ideas sobre la de los demás ptofesionales, su función económica en la sociedad capitalista, su trabajo autónomo, la omnipotencia que surge de actuar $y$ decidir sobre la vida y la muerte generan un tipo de relación particular con el resto del personal regido pot una serie de valores y actitudes específicos. En la realidad, en nuestros hospitales, el poder del médico es casi absoluto, por encima de su teal valor y razón de ser y, en contraposición, el poder de la enfermera es mínimo, por debajo de su real valor e influencia en el proceso asistencial. Este hecho es de carácter general, mundial, pero en su contemplación en España tiene innumerables posibilidades de demostración.

Como confirmación a esta afirmación, podríamos aquí enumerar un sinfín de datos objetivos. Por no hacer demasiado extensa esta ilustración, puede bastar el análisis del Real Decreto del 31-IX-1978, en el que se regula la composición de los órganos de gobierno en los hospitales. De la interpretación del mismo se deriva la siguiente distribución de representatividad de ambas profesiones:

\begin{tabular}{|c|c|c|c|c|}
\hline & Médicos & Total & Enfermeras & Total \\
\hline \multirow[t]{8}{*}{ Junta de Gobierno } & Director médico ... & 1 & & \\
\hline & Representantes del & & & \\
\hline & Centro & 6 & & 2 \\
\hline & Representantes del & & & \\
\hline & presa $\ldots \ldots \ldots \ldots \ldots$ & 12 & & 12 \\
\hline & Representantes de & & & \\
\hline & Colegios $\ldots \ldots \ldots \ldots$ & 1 & & 1 \\
\hline & $\begin{array}{l}\text { Dos miembros opta- } \\
\text { tivos } \ldots . . . . \ldots \ldots \ldots\end{array}$ & 12 & & 12 \\
\hline \multirow[t]{4}{*}{ Organos de gobierno } & Gerente...$\ldots \ldots \ldots$ & 12 & & \\
\hline & Director Médico ... & 1 & & \\
\hline & Mínimo ............. & 8 & Mínimo & 3 \\
\hline & Máximo ............ & 11 & Máximo & 5 \\
\hline
\end{tabular}


Su constatación podría carecer de trascendencia, pero en el caso de los hospitales afecta fuertemente la gestión a partir de que la misma se ha hecho compleja técnicamente y que los mismos son sujetos de minucioso análisis sobre el origen y control de sus costes. Así, la primacía del médico como profesional condiciona la gestión desde una perspectiva profesional y social.

La acción de gestión del hospital se mediatiza y en muchas ocasiones se orienta hacia la solución que conviene a la organización médica, inde. pendientemente de que ésta sea la que más convenga al resto de los trabajadores, que sea la más costosa o difícil de resolver, incluso puede decirse que en ocasiones independientemente de los intereses que más convengan al enfermo. Uno de los ejemplos sería el del horario del hospital de mafíana, como consecuencia de la práctica privada de los médicos orientada fundamentalmente por las tardes.

La profesión de la enfermera como contrapartida, no es autónoma, sino que está subordinada a las órdenes del médico, fundamentalmente en la actualidad, por desarroilarse su actividad en el seno hospitalario. Histó ricamente, existe una alta correlación entre la imagen de la enfermexía y el modelo cristiano de cuidados por una parte, y el rol de la mujer por la otra.

Es a partir del cristianismo básicamente que se asocia «cuidado del enfermo» con «gracia de Dios», por lo que hemos de considerat la im. portante infuencia de tipo religioso que opera sobre la imagen de la enfermera. Desde una perspectiva simbólica los cuidados de la enfermera aluden a la relación «materno-infantil». ${ }^{6}$

Sin embargo, el nacimiento de la enfermería como tal se remonta a la guerra de Crimea y está asociada directamente con el orden militar, dado que su acción estaba subordinada a los médicos militares. ${ }^{7}$ Nos arriesgamos a suponer que la obediencia incuestionable que el médico espera por parte de la enfermera tiene sus orígenes en esta situación, en donde el «Si, doctor» ${ }^{8}$ da una idea de la dirección en la que las órdenes van orientadas.

Podemos decir que, en general, se trata de una profesión que está desempeñada generalmente por mujeres y en la que el nivel de prestigio suele ser bajo. Hay autores que no la sitúan como profesión, sino como

6. Elvira Guilera, Revisto Rol de Enfermeria, 3 (1979).

7. R. K. Jones y P. A. Jones, «The professions and nursing: a study of the sociology of ocupations with particular to nursing profession», en Sociology in Medicine (Nueva York: A Halsted Press book-Joho Wiley and Sons, 1975).

8. Hans S. Mauksch, «Nursing: searching for a change?», pp. 206-231, en H. E. Freeman, Sol Lavine y Leo Reeder, Handbook of medical sociology (Nueva fersey: Prentice Hall, 1972). 
actividad "paramédica».' A pesar de ello, en la mayor parte de los países del mundo el personal de enfermería constituye hoy día el grupo más numeroso del personal de sanidad.

El lugar que los servicios de enfermería ocupan en los servicios de salud se debe al hecho de que, en la planificación sanitaria, el personal de enfermería se considera como un «Subsistema del sistema global de petsonal de sanidad».

Los niveles internacionalmente aceptados son: una enfermera por 5.000 habitantes y un auxiliar de sanidad por 1.000 habitantes..$^{10}$

Pero como el prestigio de la profesión disminuye, atrae a menos candidatos de calidad, por lo que se hace cada vez más difícil alcanzar estos niveles.

Para la OMS, es enfermera:

«La persona que ha terminado los estudios básicos de enfer metía y está capacitada y autorizada para asumir en su país la responsabilidad de los setvicios de enfermería que exigen al fomento de la salud, la prevención de la enfermedad y la prestación de asistencia a los enfermos.» ${ }^{11}$

Este concepto se profundiza en otros análisis efectuados a nivel de comisiones de trabajo de la OMS, que dicen:

«El papel de la enfermeta no consiste sólo en aplicar conocimientos y técnicas. Consiste en saber cuidar a las personas y, en consecuencia, en comprender a los enfermos, sus motivaciones y sus compottamientos." ${ }^{12}$

Sin embargo, a nivel de sociedad en su conjunto observamos una serie de cambios que también obligan a replantearse el status de la enfermera; ellos son:

- La evolución del concepto de salud, que pasa a ser considerado de «gracia de Dios», concepto de la beneficencia al de derecho.

9. Eliot Friedson, «La división del trabajo médico», en La profesión médica (Barcetona: Península, 1978), pp. 61-83.

10. OIT, Condiciones de trabajo de entermeria (Ginebra: Monografias, 1976).

11. OMS. Planificación de los Servicios de Entermería. Serie de Curadernos Técnicos, núm. 31.

12. OMS. Planificación de los Servicios de Enfermería. Serie de Cuadernos Técnicos, núm. 31). 
- Por otra parte, la aplicación de la ciencia y de la tecnología al área de la salud se traducen en: una mayor identificación y especialización profesional. La concreción y cesión de papeles a otras profesiones. La progresiva exigencia de los médicos de que exista una enfermería competente. El cambio de la situación de la mujer a partir de la lucha feminista también incide directamente en la profesión que es básicamente femenina.

En cualquier caso, lo que sí puede afrmarse es que, a excepción de algunos países, entre ellos «España», la profesión de la enfermera, en casos desde siempre, y en otros como resultado de su evolución, es una profesión claramente identificada, con personalidad y caracteristicas propias.

La profesión de enfermera, tal como se dice en un informe de Diana Judge en la tevista Modern Healthcare: no proporciona cuidados médicos de segunda clase y si cuidados de enfermería de primera clase.

La excepción de España la referimos a una relación de hecho significada por:

-- El propio nombre de la profesión: ayudante técnico sanitario.

- El nivel de estudios y su contenido (curriculum 1954).

- La ley que permite la convalidación automática a los médicos.

- La posición en las estructuras organizativas de los hospitales (habitualmente supeditadas al director asistencial, médico).

-- Recomendaciones como las contenidas en el informe OIT, OMS, donde, por ejemplo, España solicitó la modificación de un redactado en Ia forma siguiente:

«En la mayor parte de los países, el personal de enfermería facilita la asistencia médica», sustituirlo por: «En la mayor parte de los países, bajo la supervisión médica, el personal de enfermería.»

En cuanto a la formación del personal de enfermería, podemos decir que:

- Los estudios básicos de enfermería se consiguen mediante un programa de estudios que permite adquirir una base para el ejercicio de la profesión que luego se podrá ampliar mediante estudios superiores.

- El sistema de enseñanza básico de enfermería, inicialmente adoptado que se ha conservado en casi todas partes, es el de las escuelas finan- 
ciadas por un hospital (modelo F. Nightingale). Para más datos y conocimientos de las peculiaridades de las escuelas de ATS en España, el libro de Jesús $\mathrm{M}$. de Miguel ${ }^{13}$ constituye una fuente interesante.

- Consecuentemente, la enseñanza básica de enfermería es, en muchos casos, un adiestramiento en el servicio, lo mismo en el caso del personal titulado que en el del personal auxiliar.

- En numetosos países la formación de las enfermeras no ha evolucionado suficientemente en los últimos tiempos para preparar a las interesadas a desempeñar el nuevo papel que se le confiere en los nuevos programas sanitarios en expansión. La existencia de esta conflictiva y su planteo en España ya fue certeramente señalado en el artículo sobte la profesión médica en España de Amando de Miguel, ${ }^{14}$ y recién en la actualidad algunas escuelas adquieren el rango de universitarias.

- No obstante, en la mayoría de los casos la formación continúa dispensándose en los hospitales por el personal de los establecimientos y en función de las necesidades de los mismos. Este programa de enseñanza fue concebido únicamente en función de la asistencia directa que ha de prestarse a los enfermos, descuidando una serie de aspectos importantes de las colectividades.

Actualmente, los diplomados que han recibido su formación de base centrada en el hospital o en la entermedad (no en el concepto de salud), se encuentran limitados en sus posibilidades de actividad profesional, y a su vez, los servicios se ven limitados en cuanto a sus posibilidades de expansión y de actuación. Todo ello ha llevado a un replanteo del perfil profesional, de la planificación de tareas y por ende del contenido de la enseñanza de los mismos.

Conclusiones: Observamos entonces que existen una serie de condicionamientos que contribuyen a generar determinado tipo de estratificación en el seno del hospital. Cada una de las profesiones surge y asume posturas diferentes a lo largo de la historia que condicionan su actuación en la actualidad.

13. Jesús M. de Miguel, La reforma sanitaria en España (Madrid: Cambio 16, 1976).

14. Amando M. de Miguel: «La profesión médica en España», Papers: Revista de Sociologia, 5 (1976), pp. 117182. 
El carisma del médico, los poderes que se le confieren por ser quien decide sobre la vida y la muerte, su sexo, a veces su pertenencia a una clase social elevada, lo sitúan en una postuta supetior a la enfermera, quien por su parte pertenece en su mayoría al sexo femenino y desde los inicios, tal como ya lo señalaremos, cumple un rol subordinado. Es decir, que existen en torno a ambas profesiones una serie de ideas socio-culturales que son producto de su historia.

No obstante, la posesión de autoridad, considerada como elemento generador de la jerarquía social, no sólo proviene del legado histórico, sino básicamente de la desigual distribución del conocimiento. Siguiendo a Waitzkin, ${ }^{15}$ la estratificación ya no proviene tan sólo de la posesión o no de los medios de reproducción, cada vez más, considera que las telaciones de autoridad se han convertido en una fuente de jerarquía social. Se remite a las ideas de Dahrendorf, que postula que:

«La distribución diferencial de autoridad se convierte invariablemente en un factor determinante de los conflictos sociales que se dan de un modo sistemático, de un tipo similar a los conflictos de clase en el sentido marxista. Por lo tanto, el conflicto en el seno de un sistema social se desarrolla entre aquellos que están sometidos a esta autoridad y los que la poseen de un modo le. gítimo.s

El elemento que destaca el autor es el elemento de autonomía técnica, que plantea que tan sólo los técnicos con un nivel de formación adecuada pueden evaluar los aspectos técnicos de la medicina. La autoridad de los médicos generalmente está legitimada sobre una base «tacional» aludiendo a los postulados de Weber, por lo cual basándonos en dichos postulados, es su formación técnica, más que su autoridad carismática, la que le confiere autoridad frente a los pacientes y demás personal, en nuestro caso la enfermería.

O sea que, frente a la estratificación dada por un elemento sociocultural producto de su historia, tenemos un elemento dinámico, el nivel técnico o la formación profesional como generador de autoridad, que puede ser modificado o elevado en el caso de la enfermería, lográndose de esta manera una equiparación de autotidad en su desempeño laboral, lo que le permitiría tener el peso específico que le corresponde de acuerdo a las funciones que desempeña en el proceso asistencial, lo que podría eventual-

15. Howard B. Waitzkin y Barbara Waterman, The explotation of illness in capitalist society (Nueva York: Bobbs-Merril, 1976). 
La estratificación social a nivel hospitalario

mente producir una hipotética confrontación de autoridades, telegándose en último caso la decisión a instancias superiores.

\section{Alicia E. Kaufmann}

Departamento de Sociología

Universidad de Barcelona (Pedraibes)

Barcelona

\section{Elvira Guilera}

Escuela de Gerencia Hospitalaria

Madrid 